\title{
Integral equations for crack systems in a slightly heterogeneous elastic medium
}

\author{
A. N. Galybin ${ }^{1} \&$ S. M. Aizikovich ${ }^{2}$ \\ ${ }^{1}$ Wessex Institute of Technology, Southampton, UK \\ ${ }^{2}$ Rostov-on-Don Technical University, Russia
}

\begin{abstract}
The paper deals with derivation of a system of singular integral equations for slightly heterogeneous media. The system is derived in terms of complex potentials by introducing a small parameter by the perturbation method. The resulting system of integral equations is of the Cauchy type with the correction terms that address inhomogeneity of the media, they are presented in the form somewhat similar to the problem with body forces. Therefore the numerical methods developed for the homogeneous crack problems can directly be applied for the further numerical analysis.
\end{abstract}

Keywords: heterogeneous media, plane elasticity, singular integral equations.

\section{Introduction}

Elastic properties of many natural and artificial materials have slight fluctuations. They can be produced during formation of a material (e.g., metal sheets or concrete consolidation), loading history (rocks) or non-homogeneity of structure (ceramics). They can also be imposed for the sake to improve performance of particular materials as in fractionally graded materials, FGM. Non-homogeneity is often neglected for the determination of the fracture resistance of such materials. However, in some cases the effect induced by moduli fluctuations can create significant fluctuations of material fracture toughness, which is the case for FGM.

It should be noted that by introducing non-homogeneity of the fracture toughness one can model influence of stress fluctuations. However this case is fully not the fluctuations of material characteristics. They cannot be considered to be independent of applied load and therefore the direct superposition of two 
solutions for homogeneous and stress fluctuations may produce significant errors. A number of papers have been published on the problems dealing with the crack propagation in heterogeneous materials, e.g. [1-7]. However the case of multiple cracks in heterogeneous media has not been addressed properly. The aim of this study is to derive integral equations describing equilibrium of a system of $2 \mathrm{D}$ cracks in a slightly heterogeneous media. The latter means that the fluctuations of the elastic moduli is much less that their average values. For simplicity, the consideration is restricted to the case of a thin plate with the crack, which makes it possible to use the methods of complex variables.

The approach is based on the use of complex potentials and the perturbation technique. First a general approximate solution for the plane problem is obtained by the method developed by Vekua $[8,9]$. The form of this solution is somewhat similar to the solution presented in [10] for the case of body forces but contains two additional terms reflecting inhomogeneity. Then the system of equation is derived by the methods based on complex potentials [10] for an arbitrary smooth contour. This is a generalisation of the approach used in [7] for a single strait crack.

\section{General solution for slightly inhomogeneous media by the perturbation method}

\subsection{Equations of plane elasticity for inhomogeneous medium in complex coordinates}

The complete system of governing equations of 2D theory of elasticity consists of differential equilibrium equations (DEE), Hooke's law, strain-displacement relationships (SDR) and compatibility (of deformation) equation. By introducing complex coordinates $z=x+i y$ and $\bar{z}=x-i y$ and the following differential operators

$$
\begin{aligned}
2 \partial_{z} f & =\partial_{x} f-i \partial_{y} f, \quad 2 \partial_{\bar{z}} f=\partial_{x} f+i \partial_{y} f \\
\partial_{x} f & =\partial_{z} f+\partial_{\bar{z}} f, \quad \partial_{y} f=i\left(\partial_{z} f-\partial_{\bar{z}} f\right)
\end{aligned}
$$

one can present the governing equations as follows.

The DEE assume the form

$$
\partial \bar{z} D=\partial_{z} P
$$

where the stress functions $P$ and $D$ are used instead of the stress components $\sigma_{x x}$, $\sigma_{x y}, \sigma_{y y}$ in Cartesian coordinates

$$
P=\frac{1}{2}\left(\sigma_{x x}+\sigma_{y y}\right), \quad D=\frac{1}{2}\left(\sigma_{y y}-\sigma_{x x}\right)+i \sigma_{x y}
$$

Similarly to the stress function in (3) one can introduce the following strain function

$$
\varepsilon=\frac{1}{2}\left(\varepsilon_{x x}+\varepsilon_{y y}\right) \quad \delta=\frac{1}{2}\left(\varepsilon_{y y}-\varepsilon_{x x}\right)+i \varepsilon_{x y}
$$


and the complex displacement vectors

$$
w=u_{x}+i u_{y}
$$

where $u_{\mathrm{x}}$ and $u_{\mathrm{y}}$ are the displacement components and $\varepsilon_{x x}, \varepsilon_{x y}, \varepsilon_{y y}$ are strain components in Cartesian coordinates. Then the SDR assume the form

$$
2 \varepsilon=\partial_{z} w+\partial_{\bar{z}} \bar{w}, \quad \delta=-\partial_{z} \bar{w}
$$
form

In $2 \mathrm{D}$ the equation expressing compatibility of strain components has the

$$
2 \partial_{z \bar{z}}^{2} \varepsilon+\partial_{z z}^{2} \bar{\delta}+\partial \frac{2}{z z} \delta=0
$$

For an isotropic material the Hooke's law takes the form

$$
P=\mathrm{K} \varepsilon, \quad D=2 \mu \delta
$$

where $\mu$ is the shear modulus and $\mathrm{K}$ is a bulk modulus; for heterogeneous media they are spatial functions of coordinates.

The Lame equations (in terms of displacements) can be obtained by substitution of (8) into (2) followed by replacement of the strain functions via the SDR in the form (6). This gives

$$
\partial_{z}\left(\mathrm{~K}\left(\partial_{z} w+\partial_{\bar{z}} \bar{w}\right)\right)+4 \partial_{\bar{z}}\left(\mu \partial_{z} \bar{w}\right)=0
$$

If the problem is formulated in terms of stresses alone, then the complete system consists of the DEE (2) and the compatibility equation that is obtained by replacing the stain functions in (7) via the stress functions via the Hooke's law (8). In this case (7) assumes the form

$$
4 \partial_{z \bar{z}}^{2} \frac{P}{\mathrm{~K}}+\partial_{z z}^{2} \frac{\bar{D}}{\mu}+\partial \frac{2}{z z} \frac{D}{\mu}=0
$$

It is also possible to express the stress functions via the Airy's function and substitute it to (7), which results in a single governing equation.

It is evident that for homogeneous media $\left(\mathrm{K}=\mathrm{K}_{0}, \mu=\mu_{0}\right)$ the Lame equations take the form

$$
\partial_{z z}^{2} w+\kappa_{0} \partial \frac{2}{z z} \bar{w}=0
$$

where $\kappa_{0}=3-4 v_{0}$ for plane strain and $\kappa_{0}=\left(3-v_{0}\right)\left(1+v_{0}\right)^{-1}$ for plane stress and $v_{0}$ is homogeneous Poisson's ratio.

At the same time it is evident that both functions $P$ and $\varepsilon$ are harmonic, i.e they satisfy he Laplace equation

$$
\partial_{z \bar{z}}^{2} \varepsilon=0 \quad \partial_{z \bar{z}}^{2} P=0
$$

while the functions $D$ and $\delta$ are bi-holomorphic, i.e. their second derivatives with respect to the conjugated variable vanish

$$
\partial \frac{2}{z z} \delta=0, \quad \partial \frac{2}{z z} D=0
$$

For heterogeneous media relationships (12) and (13) are not valid. 


\subsection{Approximation for a medium with fluctuating Young's modulus}

We further consider the case when the Poisson's ratio is constant, $v_{0}$, therefore the following ratio is also constant

$$
\frac{4 \mu}{\mathrm{K}}=\kappa_{0}-1
$$

Therefore the Young modulus, E, is the only deformation characteristic that varies in space. It is assumed that these variations are much less than the average value of the Young modulus, $E_{0}=\langle E>$, over the domain considered.

Let us present the Young modulus as follows

$$
E=E_{0}+\Delta E
$$

Here the variations are designated as $\Delta E$ which is a known continuous function with respect to both variables and possessing the following property

$$
\|\Delta E\|<<E_{0}
$$

where $\|. .$.$\| stands for a norm; in particular, the norm can be defined as a global$ maximum of the function over the entire domain. Inequality (12) allows one to introduce a small parameter $\lambda$ as follows

$$
\lambda=\max |\Delta E| E_{0}^{-1}
$$

Hence (10) can be rewritten in the form

$$
E=E_{0}(1+\lambda e)
$$

where $\|e\| \leq 1$ and $0<\lambda<<1$.

Substitution of (14) and (18) into (9) results in the following equation

$$
\partial_{z z}^{2} w+\kappa_{0} \partial \frac{2}{z} z \bar{w}+\lambda\left[\partial_{z}\left(e\left(\partial_{z} w+\partial \bar{z} \bar{w}\right)\right)+\left(\kappa_{0}-1\right) \partial_{\bar{z}}\left(e \partial_{z} \bar{w}\right)\right]=0
$$

Let us further seek solution for $w$ and for all other stress and strain functions in the form

$$
Q=Q_{0}+\lambda Q_{1}+\lambda^{2} Q_{2}+\ldots
$$

where $Q$ can be $w, P, D, \varepsilon$, or $\delta$.

By substituting (20) into (19) one obtains the following recurrent process for solving the governing equation by successive approximations

$$
\begin{aligned}
& \partial_{z z}^{2} w_{0}+\kappa_{0} \partial \frac{2}{z} z \bar{w}_{0}=0 \\
& \partial_{z z}^{2} w_{j}+\kappa_{0} \partial \frac{2}{z} z \bar{w}_{j}=-F_{j-1}, \quad j=1,2, \ldots
\end{aligned}
$$

where the right hand side in the second equations in (21) has the form

$$
F_{j}=\partial_{z}\left(e\left(\partial_{z} w_{j}+\partial_{\bar{z}} \bar{w}_{j}\right)\right)+\left(\kappa_{0}-1\right) \partial_{\bar{z}}\left(e \partial_{z} \bar{w}_{j}\right)
$$

It is known from the solution at the previous iteration. 
It is evident that general solution from the zero iteration coincides with the general solution for homogeneous media presented by the KolosovMuskhelishvili formulae [10]

$$
2 \mu_{0} w_{0}=\kappa_{0} \varphi_{0}-z \overline{\varphi_{0}^{\prime}}-\bar{\psi} 0, \quad P_{0}=\varphi_{0}^{\prime}+\overrightarrow{\varphi_{0}}, \quad D_{0}=\bar{z} \varphi_{0}^{\prime \prime}+\psi_{0}^{\prime}
$$

Here $\varphi_{0}=\varphi_{0}(\mathrm{z}), \quad \psi_{0}=\psi_{0}(\mathrm{z})$ are holomorphic functions inside the domain considered and the modulus $\kappa_{0}$ is given by (14).

\subsubsection{First approximation}

Let us find the first approximation for $Q$. First let us specified the right hand side of the second equation in (21) by substituting expression from (24)

$$
F_{0}=\left(\kappa_{0}-1\right)\left[\partial_{z}\left(e P_{0}\right)-\partial_{\bar{z}}\left(e D_{0}\right)\right]
$$

Integration of the second equation in (21) can be obtained on the basis of the Pompeiu's formula [8], which has been derived for solving the nonhomogeneous Cauchy- Riemann system. In the complex variables this system can be written by a single equation of the form

$$
\partial \bar{z} w=f
$$

Solution of (24) is given by the following formula

$$
w=\phi+T f, \quad T f=-\frac{1}{\pi} \iint_{\Omega} \frac{f(\xi, \eta)}{\zeta-z} d \xi d \eta, \quad \zeta=\xi+i \eta, \quad z=x+i y
$$

where $\phi=\phi(z)$ is an arbitrary holomorphic function in the considered domain $\Omega$. This representation contains the operator $T f$ expressed by the double integral over the domain. However, formal integration of the second equation in (26) employs indefinite integrals with respect to independent variables $\bar{z}$ which gives

$$
w=\phi+\int f(z, \bar{z}) d \bar{z}
$$

Vekua [8] has demonstrated equivalence of both this presentations for analytical functions of two variables which can be expanded into power series with respect to these variables. This is a wide class of functions that covers many applications, therefore we further use indefinite integration. In the general case indefinite these integrals should be replaced by double integrals as in (25).

The general solution for $w_{1}$ can be presented as the sum of the general solution of the homogeneous equation, which has the form similar to that in (24) and a partial solution $W=W(z, \bar{z})$ of the non-homogeneous equation.

$$
2 \mu_{0} w_{1}=\kappa_{0} \varphi_{1}-z \overline{\varphi_{1}^{\prime}}-\bar{\psi}_{1}+W
$$

where new holomorphic functions $\varphi_{1}=\varphi_{1}(\mathrm{z}), \psi_{1}=\psi_{1}(\mathrm{z})$ have been introduced

After substitution of (27) into the left hand side on the second the function $W$ can be expressed in the following form

$$
\left(\kappa_{0}^{2}-1\right) W=\int\left(\kappa_{0} \bar{G}_{0}-G_{0}\right) d z, \quad G_{0}=\int F_{0} d z
$$


At the same time the stress functions presume the form

$$
P_{1}=\varphi_{1}^{\prime}+\overline{\varphi_{1}^{\prime}}+X, \quad D_{1}=\bar{z} \varphi_{1}^{\prime \prime}+\psi_{1}^{\prime}+Y
$$

Due to equilibrium the stress the functions $X$ and $Y$ are related to each other as

$$
\partial_{z} X=\partial_{\bar{Z}} Y
$$

They can also be expressed through the function $W$ as follows

$$
X=e P_{0}+\left(\partial_{z} W+\partial_{\bar{z}} \bar{W}\right)\left(\kappa_{0}-1\right)^{-1}, \quad Y=e D_{0}-\partial_{z} \bar{W}
$$

Now by introducing new holomorphic functions

$$
\varphi=\varphi_{0}+\lambda \varphi_{1}, \quad \psi=\psi_{0}+\lambda \psi_{1}
$$

one can represent the first approximation of the general solution in the form

$$
2 \mu_{0} w=\kappa_{0} \varphi-z \bar{\varphi}^{\prime}-\bar{\psi}+\lambda W, \quad P=\varphi^{\prime}+\bar{\varphi}^{\prime}+\lambda X, \quad D=\bar{z} \varphi^{\prime \prime}+\psi^{\prime}+\lambda Y
$$

Formulae (33) can be considered as a generalization of the Kolosov formulae to the case of slightly non-homogeneous medium [10]. The obtained formulas are similar to the solution for homogeneous media with body forces. They would coincide if the first terms in both expressions (31) were omitted

\section{Singular integral equations for crack problems}

\subsection{Reduction to the singular integral equation}

Let us determine boundary values of the involved functions. Since on the boundary of a domain the complex variables are not independent, this makes it possible to consider the boundary value of the function as a function of a single variable

$$
f(t)=f(t, \bar{t})=\lim _{z \rightarrow t} f(z, \bar{z}), \quad t \in \Gamma
$$

Here $\Gamma$ is the boundary of the domain, which can be considered as a union of open contours in the entire complex plane. For simplicity all the contours are assumed to be smooth, i.e. the do not contain any corner points.

The derivative of a complex valued function along the contour with respect to the complex coordinate $t$ has the form

$$
f^{\prime}(t)=\left.\partial_{z} f\right|_{z=t, \bar{z}=\bar{t}}+\left.e^{-2 i \theta} \partial \bar{z} f\right|_{z=t, \bar{z}=\bar{t}}, \quad t \in \Gamma
$$

where is $\theta=\theta(t)$ the angle between the positive direction of tangent to the contour and the positive direction of the real axis.

Let us also introduce an operator $\Delta$, that being placed before any function $f$, denote a jump of this function across the contour

$$
\Delta f(t)=f^{+}(t)-f^{-}(t)
$$

where index " \pm " corresponds to the boundary values of the function on the upper/lower surface of $\Gamma$. 
For the derivative of the complex displacements one finds

$$
2 \mu_{0} w^{\prime}(t)=\left(\kappa_{0}+1\right) \varphi^{\prime}(t)-\left(P(t)+e^{-2 i \theta} \bar{D}(t)\right)+\lambda W^{\prime}(t),
$$

Since the term in parentheses in the right hand side of (36) represent the complex vector of the applied load, $N+i T$, one can present the boundary value of the complex potential $\varphi^{\prime}(t)$ in the form

$$
\left(\kappa_{0}+1\right) \varphi^{\prime}(t)=2 \mu_{0} w^{\prime}(t)+N(t)+i T(t)-\lambda W^{\prime}(t), \quad t \in \Gamma
$$

In crack problems it is often accepted that the stress vector is continuous across the boundary, therefore $\Delta(N+i T)=0$. Bearing in mind this simplification and applying operator $\Delta$ to the both parts of (38) one finds the following expression for the jump of the sought complex potential

$$
\left(\kappa_{0}+1\right) \Delta \varphi^{\prime}(t)=2 \mu_{0} \Delta w^{\prime}(t)-\lambda \Delta W^{\prime}(t), \quad t \in \Gamma
$$

Then the potential can be found via the Cauchy integral

$$
\varphi^{\prime}(z)=\frac{1}{2 i \pi} \int_{\Gamma} \frac{\rho(t)}{t-z} d t
$$

where the unknown density $\rho(t)$ has the form

$$
\rho(t)=\frac{2 \mu_{0} \Delta w^{\prime}(t)-\lambda \Delta W^{\prime}(t)}{\kappa_{0}+1}
$$

It is evident that the expression (40) is the same as for homogeneous problem while the density contains an additional term, which is proportional to the jump of derivative of the particular solution $W$ that is known as soon as the solution of the problem for homogeneous case is known.

Once the function $\varphi^{\prime}$ is known, the stress function $P$ can be expressed in the form

$$
P=\frac{1}{2 \pi i} \int_{\Gamma} \frac{\rho(t)}{t-z} d t-\frac{1}{2 \pi i} \int_{\Gamma} e^{-2 i \theta(t)} \frac{\overline{\rho(t)}}{\bar{t}-\bar{z}} d t+\lambda X
$$

Stress function $D$ can be determined by the following way. It follows from the DEE that it can be sought in the form

$$
D=\frac{-1}{2 \pi i} \int_{\Gamma} \frac{(\bar{t}-\bar{z}) \rho(t)}{(t-z)^{2}} d t+\omega+\lambda Y
$$

where $\omega=\omega(\mathrm{z})$ is an arbitrary holomorphic function. To find this function one needs to substitute (42) and (43) into the expression for the jump of the stress vector on $\Gamma$. Since it has been assumed that this jump is zero, by the SokhotskiPlemelj formulae, [10], one should equate all non-integral terms to zero, which immediately fives the following expression for the jump of the holomorphic function, as follows

$$
\Delta \omega(t)=-\overline{\rho(t)}-\lambda \Delta Q(t), \quad Q=X+Y
$$


Then the holomorphic function $\omega(\mathrm{z})$ is determined by its jump, which eventually leads to the following expression for the stress function $D$

$$
D=-\frac{1}{2 \pi i} \int_{\Gamma} \frac{\overline{\rho(t)}+\lambda \Delta Q(t)}{t-z} d t-\frac{1}{2 \pi i} \int_{\Gamma} \frac{(\bar{t}-\bar{z}) \rho(t)}{(t-z)^{2}} d t+\lambda Y
$$

As soon as the expressions for the stress functions via the unknown density $\rho(t)$ are found one can derive a singular integral equation (SIE) of the considered problem by assuming that the stress vector is known on $\Gamma$. The final expression for the SIE can be written in the form

$$
\begin{aligned}
& \frac{1}{2 \pi i} \int_{\Gamma}\left[\frac{\rho(t)}{t-\zeta}-e^{-2 i \theta(t)} \frac{\overline{\rho(t)}}{\bar{t}-\bar{\zeta}}\right] d t+ \\
& +\frac{e^{-2 i \theta(\zeta)}}{2 \pi i} \int_{\Gamma}\left[\frac{\overline{\rho(t)}+\lambda \Delta Q(t)}{t-\zeta}+\frac{(\bar{t}-\bar{\zeta}) \rho(t)}{(t-\zeta)^{2}}\right] d t=N+i T-\lambda Q, \quad \zeta \in \Gamma
\end{aligned}
$$

The last term in the right hand side of (46) is known from the solution of the similar SIE for homogeneous domain in which it is necessary assume $W=0$ and $X=Y=0$. For a system of cracks SIE (46) can be transformed to a system of SIE, see [7] for detail.

It should be noted that in order to possess a unique solution the system (46) has to be complemented by the condition of single-valuedness of the displacements. This condition depends on the problem considered, for the case of $n$ non-intersecting cracks there should be specified $n$ additional conditions imposed in the sought function, which is similar to homogeneous case, see details in [11].

\subsection{Particular case of a straight crack}

In the case of a straight crack located on the interval $(-1,1)$ of the real axis the SIE assumes simple form

$$
\frac{1}{\pi i} \int_{-1}^{1} \frac{\overline{\rho(t)}}{t-x} d t=-[N(x)+i T(x)]+\lambda S(x), \quad|x|<1
$$

where the function $S(t)$ has been introduced as follows

$$
S(x)=Q(x)-\frac{\Delta Q(x)}{2}-\frac{1}{2 \pi i} \int_{-1}^{1} \frac{\Delta Q(t)}{t-x} d t
$$

The condition of single valuedness assume the form

$$
\int_{-1}^{1}\left[\left(\kappa_{0}+1\right) \rho(t)+\chi \Delta Q(t)\right] d t=0
$$


The solution of this problem is obtained by perturbations, see [7] for detail. It has the form

$$
\overline{\rho(t)}=\frac{i}{\pi \sqrt{1-t^{2}}}\left\{\int_{-1}^{1} \frac{\sqrt{1-x^{2}}}{x-t}[N(x)-i T(x)-\lambda S(x)] d x+C\right\}
$$

An arbitrary constant $C$ should be determined from the condition of singlevaluedness of the displacements which

It should be noted that functions $Q(t), \Delta Q(t)$ and $S(x)$ in (47)-(50) remain unknown until the problem for the homogeneous medium is solved. This solution has the form

$$
\overline{\rho_{0}(t)}=\frac{i}{\pi \sqrt{1-t^{2}}} \int_{-1}^{1} \frac{\sqrt{1-x^{2}}}{x-t}[N(x)-i T(x)] d x
$$

Here the function $\rho(t)$ is decomposed into the sum

$$
\rho(t)=\rho_{0}(t)+\lambda \rho_{1}(t)
$$

where $\rho_{0}(t)=2 \mu_{0}\left(1+\kappa_{0}\right) \Delta w^{\prime}{ }_{0}(t)$ represents a solution for the homogeneous plate

Now the function $Q(t)$ can be evaluated by substitution of the complex potential with zero sub-index in the corresponding formulae to determination of $W, X, Y, Q$ and $S$. Their explicit forms are not written here because of awkwardness.

By applying the perturbation method with the account for (52), the solution for $\rho_{1}(t)$ yields the form similar to (50) where the constant $C$ is to be determined from the condition (49). This results in

$$
C=\frac{-i \lambda}{\kappa_{0}+1} \int_{-1}^{1} \Delta Q(t) d t=0
$$

Formulae (51)-(53) present the complete solution to the problem for the straight crack in slightly non-homogeneous plate.

\section{Conclusions}

The present paper presents a system of SIE for curvilinear non-intersecting cracks in slightly non-homogeneous media in the form (46). It can be solved analytically for some simple particular cases of straight or circular cracks. For curvilinear cracks or for a system of $n$ cracks the solution can be found by any numerical procedure associated with solving a system of SIE, in particular by the methods of mechanical quadratures [11].

The obtained system can be used for analysis of the characteristics related to the fracture of heterogeneous materials and in particular to investigation of the fracture propagation in FGM. 
74 Boundary Elements and Other Mesh Reduction Methods XXXII

\section{References}

[1] F. Delale, F. Erdogan, 1988. Interface crack in a non-homogeneous elastic medium. Intt. J. Engng Sci. 26, 559-568.

[2] Y. F. Chen, F. Erdogan, 1996. The interface crack problem for a nonhomogeneous coating bonded to a homogeneous substrate. J. Mech. Phys. Solids, 44, 771-787.

[3] A.A. Al-Falou, R.C. Ball, 2000. Crack propagation in heterogeneous media. Inte Journal of Solids and Structures 37, 461-475

[4] S. Roux, D. Vandembroucq, F. Hild, 2003. Effective toughness of heterogeneous brittle materials. European Journal of Mechanics A/Solids 22, 743-749

[5] R. Luciano, J.R. Willis, 2003. Boundary-layer corrections for stress and strain fields in randomly heterogeneous materials. Journal of the Mechanics and Physics of Solids, 51, $1075-1088$.

[6] B.M. Singh, J. Rokne, J. Vrbik, R.S. Dhaliwal, 2006. Finite Griffith crack propagating in a non-homogeneous medium. European Journal of Mechanics A/Solids, 25, 867-875

[7] Galybin, A.N., 1999. An analytic solution for a straight crack in slightly non-homogeneous elastic plate Proc of Int Workshop on Fracture Mechanics and Advanced Engineering Materials (Editors L. Ye and Y-W Mai). The University of Sydney, Sydney, Australia. pp. 68-75.

[8] Vekua, I.N. 1962. Generalised analytic functions. Pergamon Press, Addison Wesley Publishing Company inc. Reading, Massachusetts, UWA.

[9] Vekua, I.N. 1967. New methods for solving elliptic equations. NorthHolland Publishing Company. John Wiley and Sons Inc. New York, UWA.

[10] Muskhelishvili, N.I. (1953) Some Basic Problems of the Mathematical Theory of Elasticity. P. Noordhoff Ltd. Groningen-Holland.

[11] Savruk, M.P. 1981. Two-Dimensional Problems of Elasticity for Bodies with Cracks. Kiev, Naukova Dumka (in Russian). 